\title{
Reply letter to "An update on direct immunofluorescence for diagnosing dermatitis herpetiformis". Could granular C3 deposits at the dermal epidermal junction be considered a marker of "cutaneous gluten sensitivity"?
}

\author{
Alice Verdelli ${ }^{1}$, Alberto Corrà², Marzia Caproni ${ }^{2}$ \\ ${ }^{1}$ Department of Experimental and Clinical Biomedical Sciences “Mario Serio", University of Florence, Florence, Italy \\ ${ }^{2}$ Section of Dermatology, Department of Health Sciences, University of Florence, Florence, Italy
}

Adv Dermatol Allergol 2021; XXXVIII (2): 346-348

DOI: https://doi.org/10.5114/ada.2021.106218

We read with interest the paper by Dmochowski et al. [1] on the direct immunofluorescence (DIF) features in dermatitis herpetiformis (DH). DIF on perilesional skin is the gold standard for $\mathrm{DH}$ diagnosis [2]. The pathognomonic finding for $\mathrm{DH}$ is a granular immoglobulin A (IgA) deposit along the dermal-epidermal junction (DEJ) with three main patterns (microgranular deposits at the tips of the dermal papillae, microgranular deposits along the DEJ, and microgranular-fibrillar/fibrillar deposits at the tips of the dermal papillae).

The authors underlined that IgA deposits at the DEJ of perilesional skin with DIF can also be found in coeliac patients with inflammatory skin diseases other than $\mathrm{DH}$. In 2007, Cannistraci et al. [3] showed the presence of granular IgA deposits in the healthy skin of patients with celiac disease (CD), who were not affected by any skin diseases, suggesting that such deposits could be considered not only a marker of DH but also, more generally, of CD. Moreover, our group reported a case-series of 6 celiac patients presenting with heterogeneous skin diseases different from $\mathrm{DH}$, characterized by a granular deposit of IgA at the DEJ and/or at the papillary tips [4]. We hypothesized that IgA deposits might be considered not only as a distinct feature of $\mathrm{DH}$ but also as an immunopathological marker of CD that could even have a diagnostic role for the disease.

These studies raise some questions about the diagnosis of DH because granular IgA deposits along the DEJ can also be found in perilesional skin of patients with $\mathrm{CD}$ with skin diseases other than $\mathrm{DH}$. This means that all the previously defined atypical DH cases could only be part of the skin spectrum associated to $C D$, rather than real $\mathrm{DH}[5]$.

The authors also reported some cases with DH-like lesions associated with C3 deposits at the DEJ. They underlined that it could be a new disease entity, different from $\mathrm{DH}$ and plausibly related to the non-coeliac gluten sensitivity (NCGS). NCGS is a syndrome characterized by intestinal and extraintestinal symptoms associated to the ingestion of gluten-containing food in subjects who are not affected by either CD or wheat allergy [6]. NCGS has been rediscovered recently, following the work published by Sapone et al. [7]. Since then, reports on NCGS have grown exponentially, as well as the number of persons treated with a gluten-free diet (GFD) because of a wide array of conditions [8]. Hashimoto et al. [9] found 20 patients with $\mathrm{DH}$-like lesions, showing granular deposition of C3 (but not IgA, IgG, or IgM) in the DEJ by DIF. They proposed the term granular C3 dermatosis (GCD) to classify these patients. In our experience, $\mathrm{DH}$-like lesions with granular C3 deposits at the DEJ have been found in NCGS patients; thus, our main hypothesis is that cutaneous gluten sensitivity (CGS), as a specific cutaneous manifestation of NCGS, may exist and could represent a diagnostic marker of NCGS [10].

To date, only a few studies have investigated the cutaneous manifestations of NCGS. Volta et al. [11] observed cutaneous involvement in $29 \%$ of patients. Faina et al. [12] found cutaneous manifestations in $65.6 \%$ of NCGS patients. Our group enrolled 17 patients with NCGS and cutaneous manifestations, with a prevalence of female sex and adulthood, highlighting that all the patients

Address for correspondence: Alice Verdelli MD, Department of Experimental and Clinical Biomedical Sciences “Mario Serio”, University of Florence, Viale Morgagni 50, 50134 Florence, Italy, e-mail alice.verdelli@hotmail.it Received: 23.03.2020, accepted: 31.05.2020. 
had skin disorders with some peculiar clinical and immunopathological aspects [10].

Patients with NCGS often showed itchy dermatoses. In up to $10 \%$ of the cases, severe itching represented the only symptom of the disease. The itching was resistant to standard therapies, but it showed prompt resolution with a GFD, with a faster reaction than in $\mathrm{DH}$ patients. Skin lesions were polymorphic, showing in the first phases an eczema-like appearance and evolving into psoriasis-like lesions in the later phases. In some cases, skin lesions overlapped the features of DH. Skin lesions were found to be predominantly located on the extensor surfaces of the upper and lower limbs and on the volar surface of the hands. Accordingly, skin manifestations in patients with NCGS usually resolved or improved significantly with a GFD.

At histopathological examination, different histopathological aspects were found, according to different clinical pictures. However, the DIF of the perilesional skin was similar in almost all the patients, showing a C3 deposit along the DEJ with a microgranular/granular pattern. This could represent a specific diagnostic marker for cutaneous lesions in NCGS patients. Over the years, many new cases of NCGS with cutaneous manifestations have been collected by our group (data not previously shown). In total, 45 patients were analysed. The median age at diagnosis was 39 years (range: $5-78$ ) with a predominant female sex (female : male ratio $=3.5: 1$ ). Itching was present in all the patients, from moderate to severe. Morphologically, the lesions were mainly erythematous, squamous, excoriated papular-vesicular, and vesicles, sometimes crusts (Figure 1). Some patients had hyperkeratotic scaly lesions, consistent with psoriasis. Lesions were located on the extensor surfaces of upper limbs (elbows and back of the hands (90\% and 18\%)), extensor surfaces of lower limbs (knees) (60\%), bottom (29\%), chest (25\%), neck (18\%), the palms of the hands $(10 \%)$, and face (6\%). At DIF, most of the patients (86.6\%) showed a C3 microgranular/granular pattern (Table 1).

Intestinal manifestations included abdominal pain (61\%), swelling (40\%), constipation (15\%), diarrhoea (20\%), and flatulence (20\%).

Symptomatic improvement was seen in all NCGS patients within an average time of 1 month after the GFD, faster than in $\mathrm{DH}$.

As Dmochowski et al. [1] suggested, we should pay attention to coining terms for apparently newly described dermatoses, but, according to our results, the introduction of the concept of specific skin manifestations related to NCGS with peculiar DIF depositions may be helpful for the management of the patients, as it is for $\mathrm{DH}$ and CD. In conclusion, gluten can be responsible for the onset of specific skin manifestations, some of which are already described as DH, while others are currently under definition, such as the CGS, immunologically characterized by the granular deposit of C3 at the DEJ. These NCGS cutaneous forms should also be considered in our clinical practice. A GFD, even in these patients, has the ability to resolve skin manifestations faster than in $C D$.

\section{Conflict of interest}

The authors declare no conflict of interest.

Table 1. Immunoreactants in direct immunofluorescence in 45 patients with non-celiac gluten sensitivity

\begin{tabular}{lccccc}
\hline & IgG & IgM & IgA & C3 & C1q \\
\hline DEJ & $0 / 45(0 \%)$ & $20 / 45(44.4 \%)$ & $0 / 45(0 \%)$ & $39 / 45(86.6 \%)$ & $15 / 45(33.3 \%)$ \\
\hline PV & $3 / 45(6.6 \%)$ & $30 / 45(66.6 \%)$ & $4 / 45(8.8 \%)$ & $33 / 45(73.3 \%)$ & $5 / 45(11.1 \%)$ \\
\hline FB & $0 / 45(0 \%)$ & $14 / 45(31.1 \%)$ & $6 / 45(13.3 \%)$ & $2 / 45(4.4 \%)$ & $2 / 45(4.4 \%)$ \\
\hline
\end{tabular}

$D E J$-dermal-epidermal junction, $P V$ - perivascular, FB-fluorescent bodies.
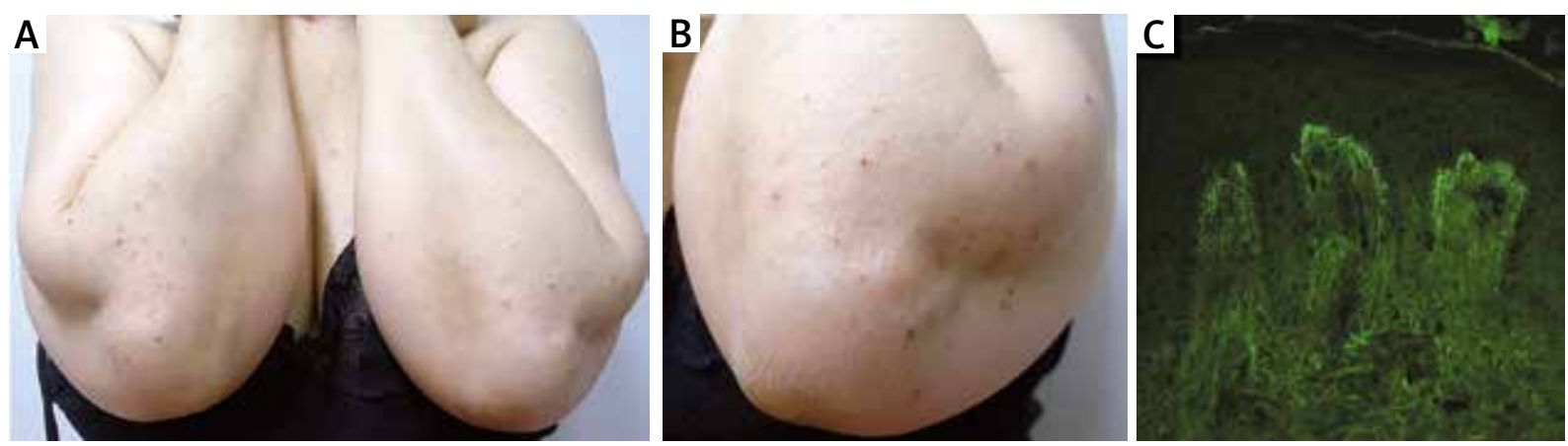

Figure 1. A-C - Skin manifestation of cutaneous gluten-sensitivity (CGS). A, B - Vescicle and erosive lesions on an erythematous base, with crusts due to scratching, located on elbows. C - Direct immunofluorescence showing granular C3 deposition along dermo-epidermal junction 


\section{References}

1. Dmochowski M, Gornowicz-Porowska J, Bowszyc-Dmochowska M. An update on direct immunofluorescence for diagnosing dermatitis herpetiformis. Adv Dermatol Allergol 2019; 36: 655-8.

2. Antiga E, Maglie R, Quintarelli L, et al. Dermatitis herpetiformis: novel perspectives. Front Immunol 2019; 10: 1290.

3. Cannistraci C, Lesnoni La Parola I, Cardinali G, et al. Co-localization of IgA and TG3 on healthy skin of coeliac patients. Eur Acad Dermatol Venereol 2007; 21: 509-14.

4. Bonciolini V, Antiga E, Bianchi B, et al. Granular IgA deposits in the skin of patients with coeliac disease: is it always dermatitis herpetiformis? Acta Derm Venereol 2019; 99: 78-83.

5. Bonciolini V, Bonciani D, Verdelli A, et al. Newly described clinical and immunopathological feature of dermatitis herpetiformis. Clin Dev Immunol 2012; 2012: 967974.

6. Roszkowska A, Pawlicka M, Mroczek A, et al. Non-celiac gluten sensitivity: a review. Medicina 2019; 55; 222.

7. Sapone A, Bai JC, Ciacci C, et al. Spectrum of gluten related disorders: consensus on new nomenclature and classification. BMC Med 2012; 10: 13

8. Lionetti E, Pulvirenti A, Vallorani M, et al. Re-challenge studies in non-celiac gluten sensitivity: a systematic review and meta-analysis. Front Physiol 2017; 8: 621.

9. Hashimoto T, Tsuruta D, Yasukochi A, et al. Granular C3 dermatosis. Acta Derm Venereol 2016; 96: 748-53.

10. Bonciolini V, Bianchi B, Del Bianco E, et al. Manifestations of non-celiac gluten sensitivity: clinical histological and immunopathological features. Nutrients 2015; 7: 7798-805.

11. Volta U, Bardella MT, Calabrò A, et al. An Italian prospective multicenter survey on patients suspected of having nonceliac gluten sensitivity. BMC Med 2014; 12: 85.

12. Faina V, Paolino G, Bavastrelli M, et al. Classification of Cutaneous manifestations in patients with non-celiac gluten sensitivity (NCGS) and wheat allergy (WA). J Am Acad Dermatol 2017; doi: 10.1016/j.jaad.2017.12.013. 